\title{
BERUFLICHE INTEGRATION VON JUGENDLICHEN IN INSTITUTIONEN DER KINDER- UND JUGENDHILFE - HERAUSFORDERUNGEN UND UNTERSTÜTZUNG
}

Karin Werner und Renate Stohler

Zürcher Hochschule für Angewandte Wissenschaften (ZHAW),

Institut für Kindheit, Jugend und Familie

E-Mail:karin.werner@zhaw.ch

URL: https://www.zhaw.ch/de/ueber-uns/person/wekal

Zürcher Hochschule für Angewandte Wissenschaften (ZHAW),

Institut für Kindheit, Jugend und Familie

E-Mail: renate.stohler@zhaw.ch

URL: https://www.zhaw.ch/de/ueber-uns/person/stol/

Zitationsvorschlag:

Werner, Karin/Stohler, Renate (2021): Berufliche Integration von Jugendlichen in Institutionen der Kinder- und Jugendhilfe - Herausforderungen und Unterstützung. In: Gesellschaft - Individuum - Sozialisation (GISo). Zeitschrift für Sozialisationsforschung, 2 (1). DOI: $10.26043 / G I S o .2021 .1 .5$

Link zum Artikel:

https://doi.org/10.26043/GISo.2021.1.5 


\title{
BERUFLICHE INTEGRATION VON JUGENDLICHEN IN INSTITUTIONEN DER KINDER- UND JUGENDHILFE - HERAUSFORDERUNGEN UND UNTERSTÜTZUNG
}

\author{
Karin Werner und Renate Stohler
}

\begin{abstract}
Der Abschluss einer Berufsausbildung ist für alle Jugendlichen eine sozialisationsrelevante Entwicklungsaufgabe. Für Jugendliche, die in Heimen leben, ist dieser Entwicklungsschritt besonders anforderungsreich. Im Artikel werden Ergebnisse einer qualitativ-explorativen Studie präsentiert, welche die Perspektive von Jugendlichen und Sozialpädagog*innen berücksichtigt. Im Zentrum stehen die Herausforderungen bei der Berufswahl, bei der Suche nach einem Ausbildungsplatz sowie die Unterstützung durch die Institutionen der Kinder- und Jugendhilfe. Die Ergebnisse zeigen, dass der Einstieg in eine Ausbildung auf Sekundarstufe II für Jugendliche in Heimen mit einer Reihe von spezifischen Herausforderungen verbunden ist, sie aber auch vielfältige Unterstützung durch die Sozialpädagog*innen erhalten.
\end{abstract}

Keywords: Berufliche Integration, Heimerziehung, Jugendliche, Care Leaver

\section{EINLEITUNG}

Der Übertritt in eine Ausbildung auf Sekundarstufe II ist für alle Jugendlichen eine bedeutsame Transition, die mit etlichen Anforderungen verbunden ist. ${ }^{1}$ Doch welche Erfahrungen machen Jugendliche, die in einer Institution der Kinderund Jugendhilfe leben bei der Berufswahl und beim Übergang von der obligatorischen Schule in eine weiterführende Ausbildung? Die Ergebnisse eines qualitativen Forschungsprojekts in den Kantonen Bern und Zürich liefern hierzu erste Antworten. Einleitend werden die Rahmenbedingungen in Bezug auf Ausbildung und Jugendhilfe in der Schweiz sowie der Forschungsstand und die Forschungsfragen präsentiert. Nach der Beschreibung des methodischen Vorgehens sowie des Samples folgen die Vorstellung und die Diskussion der Ergebnisse.

\subsection{Schule, Berufsbildung und Leaving Care in der Schweiz}

Die Verantwortung für die obligatorische Schule tragen in der Schweiz die 26 Kantone. In den meisten Kantonen beträgt die obligatorische Schulzeit 11 Jahre (Primarstufe: acht Jahre, inkl. zwei Jahre Kindergarten; drei Jahre Sekundarstufe I). Die Sekundarstufe I absolvieren die Jugendlichen auf unterschiedlichen Leistungsniveaus (EDK o. J.a). Bei Kindergarteneintritt sind die Kinder in der Regel vier und beim Abschluss der obligatorischen Schulzeit zwischen 15 und 16 Jahre alt. Bereits im Alter von 13 bis 14 Jahren wird die nachfolgende Ausbildung auf Sekundarstufe II im Rahmen des Berufswahlunterrichts aktuell, und die Jugendlichen müssen wichtige Entscheide für ihre berufliche Laufbahn fällen. Grundsätzlich gilt es zu entscheiden, ob nach der obligatorischen Schulzeit eine allgemeinbildende Ausbildung (Gymnasium, Fachmittelschule) besucht oder eine berufliche Grundbildung, umgangssprachlich Berufslehre genannt (EDK o. J.b), absolviert werden soll. Erklärtes Ziel von Bund und Kantonen ist es, dass 95 Prozent der unter 25-Jährigen einen Abschluss auf Sekundarstufe ॥ erwerben sollen (Schmidlin et al. 2018, 1). Um dieses bildungspolitische Ziel zu erreichen, wurde in den Kantonen ein vielfältiges Unterstützungsangebot für Jugendliche und junge Erwachsene entwickelt. Die meisten Jugendlichen treten nach Abschluss der obligatorischen Schule direkt in eine Ausbildung auf

\footnotetext{
${ }^{1}$ Der Begriff Sekundarstufe II bezieht sich auf qualifizierende Ausbildungen, die im Anschluss an die obligatorische Schulpflicht besucht werden, wie z. B. die berufliche Grundbildung oder weiterführende Schulen (wie Gymnasien oder Fachmittelschulen) (SKBF 2018, 2).
} 
Sekundarstufe II ein. ${ }^{2}$ In der Schweiz hat die Berufsbildung eine lange Tradition (Stalder/Clerc 2018, 339-346) und ist der populärste Bildungsweg. ${ }^{3} \mathrm{Da}$ die meisten Berufsausbildungen drei oder vier Jahre dauern, leben junge Erwachsene bis zum Ausbildungsabschluss und somit über die Volljährigkeit (mit 18 Jahren) hinaus bei den Eltern (BFS 2016, 4) und werden von ihnen finanziell sowie emotional unterstützt. Jugendliche, die nicht direkt in eine qualifizierende Ausbildung übertreten, absolvieren häufig eine Zwischenlösung (z. B. 10. Schuljahr, Praktikum). Es kommt aber auch vor, dass Jugendliche nach Abschluss der obligatorischen Schule weder eine Ausbildung beginnen noch in eine Zwischenlösung eintreten (SKBF 2018, 105).

Jugendliche, die in Institutionen der Kinder- und Jugendhilfe leben, besuchen entweder eine öffentliche oder eine heiminterne (SonderISchule. ${ }^{4}$ Auch sie sind im frühen Jugendalter mit der Berufswahl und dem Übertritt in eine qualifizierende Ausbildung auf Sekundarstufe II konfrontiert und werden in dieser Phase von den Lehrpersonen und den sozialpädagogischen Fachpersonen begleitet. Ihre Ausbildung können sie je nach Institution entweder heimextern oder auch heimintern absolvieren. ${ }^{5}$ Während Gleichaltrige - wie dargelegt - in der Regel erst nach Abschluss der Ausbildung oder noch später aus dem Elternhaus ausziehen, gestaltet sich die Situation für junge Erwachsene in Heimen diesbezüglich anders.

Jugendliche, deren Unterbringung im Heim auf dem schweizerischen Jugendstrafgesetz oder dem schweizerischen Invalidengesetz beruht, können über die Volljährigkeit hinaus im Heim wohnen bleiben (Kompetenzzentrum Leaving
Care 2020). Doch zivilrechtliche Massnahmen der Kinder- und Jugendhilfe enden in der Schweiz grundsätzlich mit Erreichen der Volljährigkeit. Es gibt bislang nur wenige Kantone (z. B. Basel-Stadt), in denen auch die Unterstützung von zivilrechtlich untergebrachten Jugendlichen über die Volljährigkeit hinaus gesetzlich verankert ist. ${ }^{6}$ In verschiedenen Kantonen existieren jedoch Bestimmungen, die einen verlängerten zivilrechtlichen Aufenthalt im Heim als „ermessensmässige Ausnahme" (Schaffner/Rein 2014, 13), höchstens aber bis 22 Jahre, ermöglichen. Mit Erreichen der Volljährigkeit stellt sich daher für zivilrechtlich untergebrachte junge Erwachsene in Heimen die Frage, ob sie nach ihrem 18. Geburtstag weiterhin im Heim wohnen können oder wohnen wollen. Care Leaver sind bei Erreichen der Volljährigkeit in der Regel noch in der Ausbildung oder haben diese noch gar nicht begonnen und befinden sich in einer Zwischenlösung. ${ }^{7}$

\subsection{Forschungsstand}

Eine Vielzahl von internationalen Studien belegt, dass Care Leaver tiefere Bildungsabschlüsse erwerben als Gleichaltrige ohne Jugendhilfeerfahrung (z. B. Courtney et al. 2009; O'Higgins et al. 2015; Köngeter et al. 2016; Cameron et al. 2018; Groinig et al. 2019). Es sind vielfältige Faktoren, welche den Bildungsweg von Care Leavern beeinflussen können. Stein (2012) verweist darauf, dass sich individuelle Belastungen der Jugendlichen, schwierige familiäre Verhältnisse, häufige Platzierungs- und Schulwechsel und unzureichende Unterstützung negativ auf die schulischen Leistungen der Jugendlichen und damit auch auf ihre weiteren Bildungswege auswirken

\footnotetext{
2 Im Jahr 2015 traten 76 Prozent der Jugendlichen nach Beendigung der obligatorischen Schulpflicht direkt in eine zertifizierende Ausbildung auf Sekundarstufe II ein (BFS 2020).

${ }^{3}$ Rund zwei Drittel der Jugendlichen entscheiden sich für die Berufslehre (SBFI 2020, 4).

${ }^{4}$ Aus Gründen der Lesbarkeit wird im Folgenden zusätzlich zum Begriff "Institutionen der Kinder- und Jugendhilfe“ synonym auch der Begriff „Heim“ verwendet; dieser entspricht der Terminologie der Jugendlichen und wird im Schweizer Kontext auch in der Praxis verwendet.

5 Jugendliche, die in einem Sonderschulheim untergebracht sind, kehren nach Abschluss der obligatorischen Schule zu ihren Eltern zurück oder treten in eine andere Institution ein.

${ }^{6}$ In verschiedenen Kantonen wird derzeit die Schaffung von entsprechenden Grundlagen geprüft oder das entsprechend revidierte Gesetz tritt demnächst in Kraft (Kanton Zürich).

${ }^{7}$ Als Care Leaver werden im Fachdiskurs Jugendliche und junge Erwachsene bezeichnet, die in Heimen oder Pflegefamilien leben und sich im Übergang ins Erwachsenenalter befinden. Entweder haben sie das Heim oder die Pflegefamilie bereits verlassen oder der Austritt steht bevor (Sievers et al. 2015, 9).
} 
können. Zudem gibt es Hinweise, dass Lehrpersonen und Sozialpädagog*innen in Bezug auf die Bildungsabschlüsse dieser Jugendlichen tiefere Erwartungen haben (z. B. Groinig et al. 2019; Hanrahan et al. 2020). Care Leaver müssen mit Erreichen der Volljährigkeit - in der Regel mit 18 Jahren - aus dem Heim oder der Pflegefamilie ausziehen, weil die Unterstützung durch die Kinder- und Jugendhilfe endet. Wie Göbel et al. (2019) darlegen, wird von Care Leavern erwartet, dass sie beim Austritt aus dem Heim bereits konkrete Pläne für ihre weitere berufliche Zukunft haben. Ihnen wird dadurch eine weniger lange Berufsfindungsphase zugestanden als Gleichaltrigen. Weitere Studien zur beruflichen Integration von Care Leavern zeigen auf, dass für den Bildungserfolg eine bedürfnisgerechte Begleitung und Unterstützung durch verschiedene Fachperson sowie die Förderung durch weitere wichtige Bezugspersonen während und nach dem Aufenthalt im Heim eine zentrale Rolle spielen (z. B. Gilligan/Arnau-Sabatés 2017; Brady/Gilligan 2019; Göbel et al. 2019; Strahl 2019; Hanrahan et al. 2020). Für die Förderung der Bildungschancen von Care Leavern sind zudem strukturelle Veränderungen, wie z. B. die Verlängerung des Heimaufenthalts über die Volljährigkeit hinaus sowie die Implementierung von Unterstützungsangeboten nach Ende der Jugendhilfe, nötig, wie verschiedene Autor*innen auf der Basis von Studienergebnissen folgern (z. B. Cameron et al. 2018; Courtney 2019; Strahl 2019; Göbel et al. 2019; Harder et al. 2020).

Für die Schweiz zeigt die Längsschnittstudie "Transition von der Erstausbildung ins Erwerbsleben (TREE)", dass die meisten der befragten jungen Erwachsenen (90 Prozent) im Alter von 30 Jahren eine nachobligatorische Ausbildung abschliessen konnten, wobei es sowohl kontinuierliche als auch diskontinuierliche Verläufe gibt (Meyer 2018, 7). Wie die Untersuchung von Schmidlin et al. (2018, XIII) von jungen Erwachsenen in der Sozialhilfe zeigt, sind es insbesondere junge Menschen mit Mehrfachbelastungen sowie diejenigen aus bildungsfernen Familien, denen es schwer fällt, eine nachobligatorische Ausbildung abzuschliessen. Für die Schweiz können zu den Ausbildungsabschlüssen von Care Leavern keine Aussagen gemacht werden, da es keine nationale Jugendhilfestatistik gibt und in den Bildungsstatistiken und -studien Care
Leaver nicht gesondert erfasst werden. Ebenso ist nicht bekannt, wie viele junge Erwachsene in der Schweiz jährlich Heime und Pflegefamilien verlassen. Hinweise darauf, dass die berufliche Integration von Care Leavern nicht immer problemlos verläuft, geben einerseits Studien zu jungen Erwachsenen in der Sozialhilfe (Schaffner 2007; Stohler/Gehrig 2014) sowie die wenigen Studien zur Thematik der Care Leaver. Letztere untersuchen die aktuelle Lebenssituation von Care Leavern aus einzelnen Institutionen (Stohler 2005) oder fokussieren einige Jahre nach Austritt retrospektiv den Lebensverlauf (Crain 2012; Schaffner/Rein 2013). Die Ergebnisse dieser wenigen Studien verweisen sowohl auf gelungene als auch auf problematische Prozesse der beruflichen Integration. Schaffner (2014) untersuchte in einem Schweizer Sonderschulheim die Deutungs- und Handlungsmuster von Fachpersonen bei der Vorbereitung von Jugendlichen auf den Übergang ins Erwachsenenalter. Es zeigte sich, dass sowohl Sonder- wie auch Sozialpädagog*innen der Berufsintegration eine hohe Bedeutung beimessen. Deutlich wird, dass insbesondere die Sonderpädagog*innen ihre Arbeit bei der Entwicklung von beruflichen Perspektiven mit dem Jugendlichen „primär als Begleitung des Abkühlungsprozesses beruflicher Aspirationen" (ebd., 58) verstehen. In einer qualitativen Befragung von Care Leavern aus einem weiteren Sonderschulheim zeigte sich, dass auch für die Jugendlichen das Absolvieren einer Ausbildung wichtig ist und dass sie die Unterstützung bei der Berufswahl rückblickend als unterschiedlich unterstützend erfahren haben (Schaffner/Läber 2017).

In der Schweiz fehlen Studien zur Berufswahl bzw. zur beruflichen Integration von Jugendlichen, die zum Zeitpunkt der Untersuchung in einem Jugendheim leben. Die nachfolgend präsentierte Studie knüpft hier an und fokussiert thematisch die Anforderungen und Unterstützung der Berufswahl und Ausbildung während des Heimaufenthalts.

\subsection{Forschungsfragen}

Die qualitative Studie „Berufliche Integration von Care Leavern in der Schweiz" untersucht die berufliche Integration von jungen Erwachsenen in Heimen im Rahmen von drei Teilstudien aus den 
Perspektiven von a) Fachpersonen aus dem Berufsbildungskontext, b) Sozialpädagog*innen und c) Jugendlichen in Heimen. ${ }^{8}$ Die Ergebnisse, die im vorliegenden Artikel präsentiert werden, beziehen sich auf die Befragungen der Sozialpädagog*innen und der Jugendlichen und fokussieren folgende Fragen: Wie erleben Jugendliche in Heimen die Berufswahl und den Übertritt von der obligatorischen Schule in eine weiterführende Ausbildung? Welchen Herausforderungen sehen sie sich gegenüber und welche Unterstützungen erhalten sie? Welche Bedeutung hat der Kontext des Heims im Zusammenhang mit der beruflichen Integration? Wie sehen Sozialpädagog*innen den Prozess der beruflichen Integration?

\section{METHODISCHES VORGEHEN}

\subsection{Sample}

Für den Feldzugang wurden in einer systematischen Recherche in den Kantonen Bern und Zürich diejenigen Heime berücksichtigt, in denen die untergebrachten Jugendlichen eine Ausbildung ausserhalb des Heims im ersten Arbeitsmarkt absolvieren. Es wurden keine Heime berücksichtigt, die heimintern oder innerhalb der übergeordneten Trägerschaft Ausbildungen in einem "geschützten Rahmen“ anbieten und/oder deren Jugendliche primär durch die Invalidenversicherung finanzierte Ausbildungen absolvieren, da wir uns im Rahmen dieser Untersuchung - vor dem Hintergrund von Überlegungen zur Chancengleichheit für Care Leaver - spezifisch für die berufliche Integration von Care Leavern in den ersten Arbeitsmarkt interessieren.

Zwölf sozialpädagogische Institutionen in den Kantonen Bern und Zürich, die aufgrund der Angebotsbeschreibung auf ihrer jeweiligen Homepage diese Voraussetzungen erfülten, wurden im Rahmen eines gezielten Sampling (Truschkat et al. 2005) für eine Teilnahme angefragt. Acht Institutionen, fünf im Kanton Zürich und drei im
Kanton Bern, erklärten sich bereit, an der Befragung teilzunehmen. Im Zeitraum von September bis November 2019 wurden für die Teilstudie 1 acht Interviews mit Sozialpädagog*innen geführt. ${ }^{9}$

Der Feldzugang für Teilstudie 2, die Befragung der Jugendlichen, erfolgte über die Sozialpädagog*innen, die an der ersten Teilstudie teilgenommen hatten. Diese dienten im Rahmen des Sampling als Gatekeeper (Petrucci/Wirtz 2007). Mit diesem Vorgehen wurde gewährleistet, dass Jugendliche erreicht werden konnten, die ihre Ausbildung ausserhalb des Heims im ersten Arbeitsmarkt absolvieren. Es bestand die Möglichkeit, sich mit anderen Jugendlichen aus derselben Institution an einer Gruppendiskussion zu beteiligen oder sich für ein Einzelinterview zu melden; damit wurde ermöglicht, dass Jugendliche sich unabhängig davon beteiligen konnten, ob in ihrer Institution eine Gruppendiskussion zustande kam oder nicht. Im Zeitraum von Dezember 2019 bis März 2020 fanden zwei Gruppendiskussionen $(G 1, G 2)$ und zwei Einzelinterviews $(\mid 1,12)$ in insgesamt vier Institutionen statt. Insgesamt elf junge Erwachsene, acht Frauen und drei Männer im Alter von 14 bis 22 Jahren, nahmen an den Befragungen teil. Zwei der Befragten besuchen noch die obligatorische Schule, fünf haben diese abgeschlossen, sind aber noch nicht in Ausbildung, sondern befinden sich in einer Zwischenlösung, und drei sind in einer Berufslehre. Eine Teilnehmende hat die Berufsausbildung abgeschlossen und das Heim kürzlich verlassen; sie nimmt als ehemalige Bewohnerin an der Gruppendiskussion teil.

\subsection{Datenerhebung und -auswertung}

Für die Befragung der Sozialpädagog*innen wurden problemzentrierte Interviews nach Witzel (2000) geführt. Die Interviews erfolgten leitfadengestützt und wurden digital aufgezeichnet. Da nicht abschätzbar war, wie viele der zwölf angefragten Institutionen sich an der Befragung beteiligen, wurde beschlossen, alle Institutionen,

\footnotetext{
${ }^{8}$ Die Studie wurde an der ZHAW Soziale Arbeit im Rahmen des ZHAW Forschungsschwerpunkts „Gesellschaftliche Integration" durchgeführt.

${ }^{9}$ Die Konzentrierung auf die Kantone Bern und Zürich ist darin begründet, dass in einem früheren Forschungsprojekt bereits Pflegekinder aus diesen Kantonen zum Leaving Care-Prozess befragt wurden und sich die Ergebnisse der beiden Befragungen somit später vergleichen lassen.
} 
die sich melden zu berücksichtigen; jedoch musste dann aus Ressourcengründen darauf verzichtet werden, alle Interviews vollständig zu transkribieren. Die Audiodateien wurden abgehört und entlang der Themen des Interviewleitfadens zusammenfassend verschriftlicht und besonders dichte Interviewpassagen wurden wörtlich transkribiert.

Die Einzelinterviews mit den Jugendlichen wurden ebenfalls als problemzentrierte Interviews nach Witzel (2000) geführt. Die Interviews erfolgten leitfadengestützt, wurden auf Tonband aufgezeichnet und vollständig transkribiert und anonymisiert. Die Gruppendiskussionen mit den Jugendlichen orientierten sich an der Vorgehensweise, wie sie von Flick (2012, 250 ff.) beschrieben wird. Das Vorgehen wurde jedoch durch einen Themenleitfaden ergänzt, der die zentralen Fragen der Untersuchung umfasste und damit die Gegenüberstellung der Ergebnisse der verschiedenen Befragungen ermöglichte. Die Gruppendiskussionen fanden in den Heimen statt und wurden digital aufgezeichnet sowie wörtlich transkribiert und anonymisiert.

Die Datenauswertung erfolgte mittels inhaltlichstrukturierender Inhaltsanalyse nach Kuckartz (2012), dabei wurden in einem mehrstufigen Auswertungsverfahren sowohl deduktive wie auch induktive Kategorien gebildet und das Befragungsmaterial in einem iterativen Prozess analysiert. Die deduktiven Kategorien wurden aus den im Interviewleitfaden bzw. Themenleitfaden enthaltenen thematischen Schwerpunkten abgeleitet; mit den induktiven Kategorien wurden latente Sinngehalte und Bedeutungen aus dem Interviewmaterial erfasst. Das Material aus den Einzelinterviews und den Gruppendiskussionen wurde integrativ ausgewertet, d.h. als Perspektive der jungen Erwachsenen zusammengeführt. Die Ergebnisse der Befragung der Sozialpädagog*innen und der jungen Erwachsenen in Heimen wurden einander anschliessend gegenübergestellt. Dabei zielte die Gegenüberstellung darauf ab, Gemeinsamkeiten und Unterschiede zwischen den beiden Perspektiven herauszuarbeiten.

\section{ERGEBNISSE}

In der Gesamtbetrachtung der Ergebnisse der beiden Befragungen fällt auf, dass der thematische Fokus in den Gruppendiskussionen und Interviews mit den Jugendlichen hauptsächlich auf der Berufswahl liegt, während bei den Sozialpädagog*innen verstärkt Fragen der Berufsausbildung thematisiert werden. Diese unterschiedliche Akzentsetzung lässt sich damit erklären, dass sich zum Zeitpunkt der Befragung sieben von elf Jugendlichen in der Phase der Berufswahl befanden. Im Folgenden werden zuerst die in den Interviews und Gruppendiskussionen angesprochenen Herausforderungen bei der $\mathrm{Be}$ rufswahl und während der Berufsausbildung vorgestellt. Daran anschliessend werden die Unterstützungen beschrieben.

\subsection{Herausforderungen bei der Berufswahl}

\subsubsection{Berufswahl zwischen Wunsch und Realität}

Die Jugendlichen und die Sozialpädagog*innen messen einer abgeschlossenen Ausbildung einen hohen Stellenwert zu, wobei darunter in den meisten Fällen das Absolvieren einer Berufslehre verstanden wird. Von den Jugendlichen wird die Möglichkeit einer weiterführenden schulischen Ausbildung auf Sekundarstufe II nur einmal erwähnt. Auch die sozialpädagogischen Fachpersonen thematisieren keine allgemeinbildenden Ausbildungsgänge, wie der Besuch von gymnasialen Maturitätsschulen oder Fachmittelschulen. Sie weisen darauf hin, dass Jugendliche manchmal ihre "unrealistischen Berufswünsche“ den Möglichkeiten anpassen müssen, wenn eine Diskrepanz zwischen dem Wunschberuf und der Realität des Arbeits- und Ausbildungsmarktes besteht. Oder wenn die Leistungsfähigkeit und Belastbarkeit des Jugendlichen nicht den Anforderungen des Wunschberufes entspricht, wie dies eine Sozialpädagogin formuliert:

"Im Moment ist es eine konkrete Herausforderung, die Jugendlichen auf das Niveau heranzuführen, das sie eigentlich bewältigen können. Also von überhöhten Vorstellungen und Erwartungen runterholen und zur Einsicht zu führen, dass sie jetzt zum Beispiel eine PRA-Ausbildung [Praktische Ausbildung, Anm. d. Verf.] machen 
können und nicht mehr, einfach von der schulischen Leistungsfähigkeit und von der Belastbarkeit her."

Eine Fachperson thematisiert die generell niedrigen Bildungserwartungen der Sozialpädagog*innen an die Jugendlichen und kritisiert, dass Jugendliche in Heimen tendenziell in weniger anspruchsvolle Schnupperlehren und Berufslehren beraten werden. Begründet wird dies damit, dass sozialpädagogische Fachpersonen den Jugendlichen bei der Berufswahl zu wenig zutrauen und deshalb eher auf Nummer sicher gehen möchten.

\subsubsection{Entscheidungsfindung und früher Zeit- punkt der Berufswahl}

Die Berufswahl stellt eine Herausforderung dar, die von den Jugendlichen in allen Interviews und Gruppendiskussionen ausführlich besprochen wird. Sie erzählen, dass es schwierig ist zu wissen, welchen Beruf man erlernen möchte, und begründen die Schwierigkeiten bei der Entscheidungsfindung unter anderem mit dem Zeitpunkt der Berufswahl, der im dualen Bildungssystem der Schweiz nach der Sekundarstufe I relativ früh beginnt, wie beispielsweise Anita ausführt:

"Ich finde, man kommt einfach recht schnell da rein. Also man muss ja schon mit irgendwie 14 oder 15 wissen, was man lernen will, und das ist halt ein wenig stressig." (G2)

\subsubsection{Fehlende Unterstützung von der Familie}

Die Sozialpädagog*innen sehen die fehlende Unterstützung von der Familie und manchmal auch fehlende elterliche Vorbilder in Bezug auf Berufsausbildungen und Berufstätigkeit als problematisch. Auch die Jugendlichen sind der Ansicht, dass innen Vorbilder fehlen und sie wenig Unterstützung von den Eltern erfahren. Einige thematisieren, dass familiäre Probleme sie bei der Suche nach einer Lehrstelle behindern würden, wenn sie noch zu Hause bei ihren Eltern lebten. Aber auch das Unvermögen der Eltern, ihnen in schulischen Angelegenheiten zu helfen, oder die Arbeitslosigkeit der Eltern werden angesprochen, wie die Aussage von Anita verdeutlicht:
„Ich glaube, bei mir macht es einen sehr grossen Unterschied, weil wäre ich zuhause, würde ich, glaube ich, nicht viel einsetzen für meine Zukunft [...]. Meine Mam, die arbeitet ja auch nicht." (G2)

\subsubsection{Stigmatisierung als „Heimkind“}

Einige Jugendliche fühlen sich als "Heimkind“ stigmatisiert und bei der Lehrstellensuche benachteiligt. Sie erzählen von negativen Zuschreibungen auf Grund der Wohnform und der psychischen Gesundheit. So werde man als „Heimkind" automatisch als "Problemkind“ stigmatisiert, das die geforderten Leistungen während der Ausbildung vermutlich nicht erbringen oder sonst Probleme machen werde. Benachteiligungen bei der Lehrstellensuche auf Grund von Stigmatisierungen ist ein Aspekt, der insbesondere in einer Gruppendiskussion von den Jugendlichen rege besprochen und thematisch vertiefend behandelt wird. Sophie führt dazu aus:

„Also so, ich denke, das ist immer noch mega krass, wie Ausbildungsbetriebe, egal aus welchen Gründen man jetzt nicht mehr Zuhause ist, da stigmatisieren sie uns: ,Ah, das ist ein Problemkind'." (G1)

Jugendliche, die der Ansicht sind, dass das Heim als Adresse ein Hindernis bei der Lehrstellensuche ist, entwickeln unterschiedliche Strategien, wie sie damit umgehen. Das kann bedeuten, dass die Wohnsituation bei Bewerbungen zunächst verheimlicht wird, um nicht auf Grund von Vorurteilen stigmatisiert zu werden. So erzählt z. B. Marie von ihrem Vorgehen bei der Suche nach einer Berufslehre:

"Ich bin auch zum Bewerbungsgespräch gegangen. [...] Dort habe ich es auch wieder nicht gesagt und dann, ganz normal, dann haben sie mich wie genommen. Und dann, am Schluss [im Bewerbungsverfahrensprozess, Anm. d. Verf.], als es um die Finanzierung gegangen ist, habe ich es gesagt", [dass sie im Heim lebt, Anm. d. Verf.]. (G1)

Die Wohnform im Heim als Stigma und Benachteiligung bei der Lehrstellensuche kommt auch bei den sozialpädagogischen Fachpersonen zur Sprache. So erzählt beispielsweise ein Sozialpädagoge: 
„Ich habe ein paar Mal Jugendliche erlebt, die schnuppern gegangen sind und gar nichts gesagt haben, weil sie nicht von ihren Problemen erzählen wollen, am Anfang, sondern weil sie als normal, als normal funktionierende Person wahrgenommen werden wollen".

Die Jugendlichen sprechen im Zusammenhang mit den Stigmatisierungserfahrungen auch über die Fremdeinschätzung ihrer Belastbarkeit. Dabei kritisieren sie, dass sie auf Grund der Wohnsituation im Heim als nicht ausreichend psychisch stabil eingeschätzt werden und oft auf eine labile Persönlichkeit geschlossen wird, was ihre Chancen bei der Lehrstellensuche mindert. Zum anderen reflektieren die jungen Erwachsenen die eigene Belastbarkeit aber auch sehr offen. So interessieren sich einige junge Frauen für Berufe in der Betreuung oder Pflege, die eine hohe psychische Belastbarkeit erfordern. Gleichzeitig äussern aber einige von innen Zweifel daran, ob sie diesen hohen Anforderungen gerecht werden können, wenn z. B. die eigene Erfahrung gezeigt hat, dass die psychische Stabilität Schwankungen unterliegt. Dies kann bezogen auf die Berufswünsche zu Dilemmas führen, wie die Aussage von Stella zeigt:

„Das finde ich halt auch noch schwierig. Wenn so bei Lehrstellen, wenn du jetzt irgendwie, wie nicht richtig belastbar bist, aber du doch eine Lehrstelle finden musst und du gehst zu einem Beruf, der dich mega interessiert, aber, aber du brauchst psychische Belastbarkeit, aber du hast das irgendwie, irgendwie auch nicht, dann ist das irgendwie auch voll blöd, obwohl dich eigentlich der Beruf mega interessiert." (G1)

\subsection{Herausforderungen während der Berufsaus- bildung}

\subsubsection{Mehrfachbelastungen}

Neben Herausforderungen, die alle Jugendlichen während einer Berufsausbildung bewältigen müssen, wie Leistungsanforderungen und Rollenerwartungen im Betrieb und in der Berufsschule, kommen laut den Sozialpädagog*innen bei jungen Erwachsenen in Heimen viele weitere Belastungen hinzu, wie z. B. private und familiäre Probleme, geringe Konzentrationsfähigkeit und fehlende Energie.
3.2.2 Anforderungen durch die spezielle Wohnsituation im Heim

Die Jugendlichen erzählen von den Anforderungen, die aus der speziellen Wohnsituation im Heim resultieren; dass sie beispielsweise viele zusätzliche Termine wie Gruppenabende, Gespräche mit der Bezugsperson, Therapiesitzungen etc. koordinieren müssen. Sophie erläutert:

„Und das kostet so viel Zeit. Auch Ausbildung machen, dort funktionieren, und hier muss man ja auch irgendwie funktionieren. [...] Das ist so ein wenig, man hat eine doppelte Belastung, die man zuhause in dem Sinn nicht hätte." (G1)

Auch den sozialpädagogischen Fachpersonen sind die zusätzlichen Anforderungen durch die Wohnsituation im Heim bewusst, sie werden allerdings weniger ausgeprägt in den Blick genommen. Neben den bereits von den Jugendlichen genannten Aspekten wird zusätzlich das Bewahren der Konzentration trotz Ablenkung durch Mitbewohner*innen genannt.

\subsection{Unterstützung während der Berufswahl}

\subsubsection{Unterstützung durch die obligatorische Schule}

In Bezug auf die Berufswahl wird die obligatorische Schule von den Jugendlichen als unterstützend wahrgenommen, wobei insbesondere der Berufswahlunterricht positiv hervorgehoben wird. Bei einigen haben zudem einzelne Lehrpersonen einen grossen Einfluss auf die Berufswahl ausgeübt, in dem sie Jugendliche persönlich auf konkrete Berufe hingewiesen haben, die sie für die Betroffenen als passend erachteten, wie beispielsweise die Aussage von Alex zeigt:

„Und dann hat mir mein Lehrer halt ein Blatt in die Hand gedrückt und gemeint: ,Das könnte vielleicht noch zu dir passen. 'Ist, das ist dann halt der Hochbauzeichner gewesen." (I1)

\subsubsection{Sozialpädagogische Unterstützung im Heim}

Die Sozialpädagog*innen nennen eine Reihe von Unterstützungsleistungen, die sie in der Rolle als Bezugsperson eines Jugendlichen erbringen, wie etwa die Begleitung bei Terminen im Berufs- und 
Informationszentrum, Hilfe bei der Suche von Schnupperlehren und beim Schreiben von Bewerbungen. Aus Sicht der Jugendlichen ist vor allem die Unterstützung beim Erstellen von schriftlichen Bewerbungsdossiers zentral. Das professionelle und teilweise auch private Netzwerk der Sozialpädagog*innen spielt bei der beruflichen Integration eine grosse Rolle. Sowohl Jugendliche als auch Fachpersonen heben die Vernetzung von Mitarbeitenden mit einzelnen Ausbildungsbetrieben hervor. Einige Sozialpädagog*innen kennen eine ganze Reihe von Betrieben, mit denen sie in den vergangenen Jahren beruflich oder privat Kontakt hatten und die sie für Ausbildungsplätze für einzelne Jugendliche kontaktieren können. Auch weitere Mitarbeitende des Heims können eine wichtige Ressource sein, wie beispielsweise die Aussage von Luis zeigt, der über die Vermittlung der Köchin des Heims zu einer Lehrstelle in einem externen Betrieb gekommen ist:

„Unsere Köchin hat natürlich Bezug gehabt, weil sie immer geliefert haben. Sie hat sich mit dem Chef voll gut verstanden." (I2)

\subsubsection{Heim als Ermöglichungsraum}

Ein Aspekt, den die Jugendlichen explizit hervorheben, ist das Heim als Raum, der es innen ermöglicht, sich ungestört der Berufswahl widmen zu können. Die problematische familiäre Situation im Elternhaus und die damit verbundenen Ablenkungen erschweren für viele Jugendliche die Konzentration auf die Berufswahl. Im Heim dagegen finden sie einen Raum, in dem sie sich auf die eigenen entwicklungsspezifischen Aufgaben fokussieren können, wie die Ausführungen von Nikki illustrieren:

„Also für mich hat es einen Einfluss, weil wenn ich jetzt zuhause wäre, ich hätte wirklich gar keine Zeit oder ich würde die ganze Zeit, also ich bin gestört gewesen. Ich wäre gestört von meiner Familie und nachher hätte ich einfach gar keine Zeit, zum Bewerbungen schreiben." (G2)

\subsection{Unterstützung während der Berufsausbil- dung}

3.4.1 Hilfe von sozialpädagogischem Team und Bezugsperson

Während der Phase der Berufsausbildung leisten sozialpädagogische Teams einen wichtigen Beitrag, indem sie die Jugendlichen während der Ausbildung immer wieder motivieren und sie beim Erlernen von relevanten Fähigkeiten wie beispielsweis Pünktlichkeit oder Zuverlässigkeit fördern. Auch Lernabende in den Wohngruppen oder individuelle Hausaufgabenhilfe werden angeboten. Aus Sicht der Jugendlichen ist vor allem die Hilfe, die sie zur Bewältigung der schulischen Anforderungen erhalten, sehr wichtig.

Die Sozialpädagog*innen heben die individuelle Unterstützung durch die Bezugsperson hervor, die mit den Jugendlichen regelmässig Fragen und Schwierigkeiten der Berufsausbildung bespricht. Die Begleitung durch die Bezugsperson wird auch von den Jugendlichen als besondere Hilfestellung hervorgehoben, wie beispielsweise Alex ausführt:

„Ja, ich werde immer gefragt, ob es läuft, ob es gut ist, es gibt auch wöchentlich eine Sitzung, also ein Gespräch." (I1)

Die Bezugsperson steht je nach Bedarf auch in einem mehr oder weniger regelmässigen Austausch mit dem Ausbildungsbetrieb und/oder der Berufsfachschule. Insgesamt kommt zum Ausdruck, dass die Jugendlichen spüren, dass die sozialpädagogischen Fachpersonen der Berufsausbildung einen grossen Stellenwert beimessen und sich die Bezugsperson für ihre berufliche Entwicklung interessiert und engagiert.

\subsubsection{Externe Unterstützungsangebote}

Die Sozialpädagog*innen weisen auf weitere, heimexterne Hilfen hin, wie Lernunterstützungsangebote der Berufsfachschulen, individuell organisierten Nachhilfeunterricht oder Berufsinformationszentren. 
3.4.3 Wenig Unterstützung aus dem sozialen Umfeld

Insgesamt wird die Unterstützung der Eltern während der Berufswahl und Berufsausbildung von den Sozialpädagog*innen als gering wahrgenommen. Nur bei einzelnen Jugendlichen wird die Ausbildung durch die Mutter oder den Vater aktiv gefördert und begleitet, indem diese $z$. B. an wichtigen Gesprächen teilnehmen. Bei einigen Jugendlichen wirkt die Familie aus ihrer Perspektive unterstützend auf den Ausbildungsprozess, bei anderen ist sie jedoch eher ein Hindernis oder Störfaktor und die familiären Probleme erschweren es den Jugendlichen, sich auf die Ausbildung zu konzentrieren.

Die Sozialpädagog*innen weisen auf mangelnde unterstützende Kontakte der Jugendlichen ausserhalb der Institution hin. Den meisten Jugendlichen fehlen Personen, die sie unterstützen, an sie glauben und innen etwas zutrauen. Nur vereinzelt erleben Sozialädagog*innen das weitere soziale Umfeld als fördernd, wie etwa einen Paten oder eine Patin oder Beistandspersonen, die sich besonders für die Ausbildung einer oder eines Jugendlichen engagieren und sie oder ihn ermutigen. Es ist auffallend, dass auch nur einzelne Jugendliche von Unterstützungen ausserhalb des Heims berichten.

\subsubsection{Krisenbewältigung}

Aus der Perspektive der Sozialpädagog*innen ist ihr Einsatz während Krisen in der Ausbildung besonders wichtig. Sie ermutigen die Jugendlichen in Gesprächen und es werden runde Tische mit allen relevanten Beteiligten wie Ausbildner*innen, Lehrpersonen, Eltern und Beistandspersonen organisiert. Ziel dieser vermittelnden Gespräche ist es, Klärungen vorzunehmen und einen Ausbildungsabbruch zu verhindern. Doch nicht immer ist dies möglich; manchmal kommt es trotz aller Unterstützung zu einem Lehrabbruch. In diesen Situationen helfen die Fachpersonen den Jugendlichen, die Lehre in einem anderen Ausbildungsbetrieb fortzusetzen oder die Ausbildung auf einem tieferen Anspruchsniveau abzuschliessen, wenn beispielsweise vorwiegend schulische Probleme im Vordergrund stehen. Manchmal ist auch eine erneute Phase der Berufswahl angezeigt, damit die Jugendlichen eine für sie passendere Ausbildung beginnen können.

\section{5 Übergang in die Selbständigkeit}

\subsubsection{Zeitliche Parallelität von Ausbildungsab- schluss, Stellen- und Wohnungssuche}

In fast allen befragten Institutionen endet spätestens mit dem Ausbildungsabschluss auch die Finanzierung der stationären Jugendhilfe und die jungen Erwachsenen müssen das Heim zu diesem Zeitpunkt verlassen. Für die Sozialpädagog*innen ist es deshalb eine zentrale Aufgabe, zusammen mit ihnen rechtzeitig eine geeignete Wohnmöglichkeit zu suchen. Da sich die Jugendlichen in dieser Phase prioritär auf die Lehrabschlussprüfung konzentrieren, übernehmen in einigen Institutionen die Sozialpädagog*innen die Suche nach einer geeigneten Wohnmöglichkeit. Neben der Wohnungssuche ist es auch Aufgabe der Bezugsperson, die jungen Erwachsenen darin zu unterstützen, eine Arbeitsstelle zu suchen, wenn sie nach dem Ausbildungsabschluss nicht im selben Betrieb weiterarbeiten können oder wollen. Es wird deutlich, dass das zeitliche Zusammenfallen des Ausbildungsabschlusses und die Beendigung der stationären Hilfe für die jungen Erwachsenen ein markanter Einschnitt und anspruchsvoller Schritt ist, der im Hinblick auf einen gelingenden Übergang in ein selbständiges Leben aus Sicht der sozialpädagogischen Fachpersonen besonders intensiv begleitet werden muss.

\subsubsection{Unsicherheit und Orientierungslosigkeit}

"Später habe ich ja auch keine Hilfe" (G1), sagt Rita in der Gruppendiskussion. Die Vorstellung des Austritts aus dem Heim löst bei einigen Jugendlichen Unsicherheit und Orientierungslosigkeit aus. Sie äussern ihre Sorge, dass ihnen nach dem Austritt für Fragen der beruflichen und persönlichen Entwicklung keine Ansprechpersonen mehr zur Verfügung stehen könnten und sie bei Problemen keine Unterstützung mehr erhalten.

Zwei Jugendliche aus dem Sample haben bereits einen Heimaustritt erlebt. Dieser wird von ihnen als ein einschneidender biographischer Moment 
geschildert. Sophie, die als ehemalige Bewohnerin an der Gruppendiskussion teilnimmt, schildert ihre Erfahrungen:

"Man bereitet sich wirklich auf das Nachher vor, weil eigentlich ist es nicht schwierig hier zu sein. [...] Aber nachher ist es viel schwieriger." (G1)

Trotz der Vorbereitung sind die beiden Jugendlichen nach dem Heimaustritt mit unerwarteten Schwierigkeiten konfrontiert gewesen, wie z. B. den vielen administrativen Aufgaben und dem plötzlichen Alleinsein.

\subsection{Berufliche Weiterentwicklung}

\subsubsection{Zukunftspläne}

Die berufliche Entwicklung endet nicht mit dem Lehrabschluss, denn heute werden oft bereits kurze Zeit nach Ausbildungsabschluss berufliche Weiterbildungen angestrebt. Auch die befragten Jugendlichen haben berufliche Zukunftspläne. Die meisten möchten nach Ausbildungsabschluss auf dem erlernten Beruf weitere Arbeitserfahrungen sammeln, Geld verdienen und damit kleinere Pläne wie Anschaffungen oder Reisen realisieren. Ein Grossteil der Jugendlichen macht sich auch Überlegungen zur mittelfristigen beruflichen Entwicklung. Einige möchten sich innerhalb des Berufsfeldes, in welchem sie die Ausbildung absolviert haben, weiterentwickeln. Andere fassen die Möglichkeit einer Zweitausbildung (z. B. andere Lehre), eine weiterführende Schule (z. B. Berufsmatura) oder ein Studium (höhere Fachschule, Fachhoch- oder universitäres Studium) ins Auge, wie das Beispiel von Anita zeigt:

„Ja nachher, wenn ich die Lehre fertig habe, kann ich ja dann noch überlegen, ob ich noch die BM [Berufsmatur, Anm. d. Verf.] machen will oder irgendwie Fachhochschule oder Weiterbildung oder halt so." (G2)

Eine andere junge Frau, die zurzeit eine Lehre als Fachangestellte Gesundheit absolviert, möchte beispielsweise später gerne Medizin studieren. Insgesamt wird aber auch spürbar, dass die Jugendlichen unsicher sind und Zweifel haben, ob solche weiterqualifizierenden Berufsausbildungen für sie realistisch sind. Insbesondere die
Frage der Finanzierung von weiteren beruflichen Plänen beschäftigt sie.

\subsubsection{Erschwerte Bedingungen für Care Leaver}

Einige Sozialpädagog*innen machen darauf aufmerksam, dass junge Erwachsene, die in Heimen leben bzw. gelebt haben, erschwerte Bedingungen haben, Weiterbildungen oder Zweitausbildungen zu absolvieren, wie die Aussage einer Sozialpädagogin veranschaulicht:

„Ein Jugendlicher, der das KV [Kaufmännische Ausbildung, Anm. d. Verf.] abgeschlossen hat, möchte eigentlich etwas ganz anderes, eine andere Ausbildung machen, und das wird schwierig werden, denn es wird inn niemand finanzieren. Da sind wir nun am Klären, was gäbe es denn für Möglichkeiten."

Die Finanzierung von Zweitausbildungen oder Weiterbildungen ist für junge Erwachsene, die das Heim verlassen, eine besondere Herausforderung. Häufig sind die leiblichen Eltern nicht in der Lage, sie finanziell zu unterstützen, und das Realisieren von beruflichen Zukunftsplänen, z. B. eine Weiterbildung, ist deshalb für sie mit zusätzlichem Aufwand verbunden, sich Finanzierungsmöglichkeiten zu erschliessen. Einige Care Leaver ziehen deshalb Weiterbildungen oder weiterführende Ausbildungen (z. B. ein Fachhochschulstudium) gar nicht in Betracht.

\subsection{Fehlendes Wissen über berufliche Weiter- entwicklung der Care Leaver}

Den Sozialpädagog*innen ist nur wenig über den weiteren Lebensverlauf der ehemaligen Heimbewohnenden bekannt. Die Informationen stammen aus zufälligen Begegnungen mit Care Leavern, weil sie an Ehemaligen-Veranstaltungen teilnehmen oder der Institution einen Besuch abstatten. Das Wissen, das aus diesen sporadischen und zufälligen Begegnungen resultiert, wird von den Fachpersonen relativierend eingeschätzt, insofern innen bewusst ist, dass sich daraus kein umfassendes Bild der beruflichen und persönlichen Entwicklung der Care Leaver erschliessen lässt, wie die Aussage eines Sozialpädagogen verdeutlicht: 
„Wir machen einen Tag der offenen Tür, an dem ehemalige Jugendliche kommen. Das Problem ist natürlich, es kommen genau diejenigen, die es geschafft haben, die erfolgreich sind. Oder wenn sie ein neues Auto haben, (lacht) kommen sie, es zeigen. Und bei den anderen, wo es vielleicht aus beruflicher Reflexion spannender wäre, dort verliert sich halt der Kontakt oft."

\section{SCHLUSSFOLGERUNGEN UND DISKUS- SION}

Aufgrund des qualitativ explorativen Forschungsdesigns können die Ergebnisse der vorliegenden Studie nicht verallgemeinert werden. So muss in Bezug auf die Repräsentativität des Samples angemerkt werden, dass dieses nur Jugendliche und Sozialpädagog*innen aus Institutionen in zwei Kantonen umfasst. Doch die Studie gibt Hinweise auf wichtige Aspekte im Zusammenhang mit der beruflichen Integration von jungen Menschen, die in Heimen leben. Zusammenfassend zeigen die präsentierten Ergebnisse, dass sich den Jugendlichen bei der Berufswahl und während der Ausbildung etliche Herausforderungen stellen, sie jedoch auch vielfältige Unterstützung durch die Sozialpädagog*innen und durch weitere Fachpersonen erhalten.

\subsection{Formaler Bildungsabschluss unter erschwer- ten Bedingungen}

Schweizer Jugendliche müssen bereits im Alter von 13 bis 14 Jahren relevante Entscheidungen für ihre Bildungslaufbahn treffen. Für Jugendliche, die in Heimen leben, kann diese Phase eine besondere Herausforderung darstellen, wie die Ergebnisse zeigen. So beschreiben die jungen Frauen und Männer - wie andere Jugendliche auch - die Berufswahl als anspruchsvoll. Dieser Befund kann, wie einige Jugendliche auch betonen, darauf zurückgeführt werden, dass für die Bildungslaufbahn relevante Entscheide in der Schweiz sehr früh getroffen werden müssen.

Die Themen Berufswahl, Lehrstellensuche und Ausbildung beschäftigen die Jugendlichen stark. Der Erwerb eines Ausbildungsabschlusses ist ein wichtiges Ziel der befragten Jugendlichen. Sie orientieren sich damit an der in der Schweiz gültigen Norm, im Anschluss an die obligatorische
Schulzeit eine Ausbildung auf Sekundarstufe II zu absolvieren. Dieser Befund stimmt mit den Ergebnissen der Studien von Schaffner und Läber (2017) sowie von Mangold und Rein (2014) überein, die darlegen, dass ein Ausbildungsabschluss zum Normalitätsverständnis von fremduntergebrachten Jugendlichen gehört.

Die Situation der Jugendlichen im untersuchten Sample macht deutlich, dass sie auf dem Weg zu einem formalen Abschluss aufgrund ihrer familiären Situation und damit verbundenen individuellen Belastungen mit mehr Anforderungen konfrontiert sind als Gleichaltrige, die bei ihren Eltern leben. Gleichzeitig erhalten sie weniger Unterstützung durch ihre Familien und aus ihrem sozialen Umfeld. Neben der Berufswahl und weiteren entwicklungsrelevanten Aufgaben, müssen Anforderungen im Heim bewältigt werden, z. B. Teilnahme an Gruppenabenden und Standortgesprächen wie auch andere Studien zeigen (z. B. Stein 2012; Groinig et al. 2019). Ein weiteres Ergebnis der präsentierten Studie zeigt, dass Sozialpädagog*innen vielfältige Unterstützung leisten, damit Jugendliche einen formalen Abschluss erreichen können. Sie begleiten die Jugendlichen bei der Bewerbung um Lehrstellen, während der Ausbildung und auch bei der Stellen- und Wohnungssuche nach Ausbildungsabschluss. Neben der Unterstützung durch die individuelle Bezugsperson spielen die Netzwerke von Sozialpädagog*innen und weiteren Mitarbeitenden im Heimkontext eine wichtige Rolle bei der Berufswahl und der Ausbildungsplatzsuche. Darüber hinaus hat die Unterstützung durch die Sozialpädagog*innen vor allem während Krisen in der Ausbildung eine zentrale Funktion. Die grosse Bedeutung der sozialpädagogischen Unterstützung im Zusammenhang mit der beruflichen Integration heben auch andere Autor*innen (Gilligan/Arnau-Sabatés 2017; Strahl 2019; Göbel et al. 2019) hervor.

\subsection{Stigmatisierungserfahrungen}

Aus der präsentierten Untersuchung geht weiter hervor, dass die Jugendlichen bei der Suche nach einem Ausbildungsplatz aufgrund ihrer Unterbringung im Heim Stigmatisierungserfahrungen machen. So zeigen die Gespräche mit den jungen Erwachsenen, dass sie während der 
Lehrstellensuche auf gewisse Ausbildungsverantwortliche treffen, die mit im Heim lebenden Jugendlichen Probleme assoziieren oder ihnen geringere psychische Belastbarkeit zuschreiben und sie damit stigmatisieren. Stigmatisierungserfahrungen von fremduntergebrachten Kindern und Jugendlichen werden auch von anderen Forschenden thematisiert (z. B. Rogers 2017; Groinig et al. 2019; Strahl 2019; Rein 2020). Um dem Stigma „Heimkind“ entgegen zu wirken, entwickeln die befragten Jugendlichen unterschiedliche Normalisierungsstrategien, indem sie z. B. den Wohnort Heim im Bewerbungsverfahren so lange wie möglich verheimlichen. Normalisierungsbemühungen von Care Leavern und Pflegekindern werden in etlichen aktuellen Studien thematisiert (Rein 2020; Groinig et al. 2019; Werner 2019; Reimer 2017).

In den präsentierten Ergebnissen wird aber auch deutlich, dass das Heim von den Jugendlichen nicht ausschliesslich als Ort wahrgenommen wird, der Stigmatisierungserfahrungen hervorruft. So verweisen sie auch auf die Funktion des Heims als Raum, der es ihnen ermöglicht, sich auf ihre berufliche und persönliche Entwicklung zu konzentrieren, wie dies beispielsweise auch Strahl (2019) in einer qualitativen Studie herausgearbeitet hat.

\subsection{Geringere Chancen für berufliche Weiterent- wicklung}

Die präsentierten Ergebnisse zeigen, dass bei allen Befragten der Abschluss einer Berufslehre als nächstes wichtiges Ziel im Vordergrund steht. Auffallend ist, dass sowohl von den Jugendlichen wie auch von den Sozialpädagog*innen die Möglichkeit einer allgemeinbildenden weiterführenden Schule, wie z. B. das Gymnasium, nur einmal erwähnt wird. Dieses Ergebnis kann unterschiedlich interpretiert werden. So kann einerseits vermutet werden, dass sich die befragten Jugendlichen und Sozialpädagog*innen am meist gewählten Bildungsweg in der Schweiz der Berufslehre - orientieren und diesen für erstrebenswert halten. Andererseits kann das Ergebnis als Hinweis gedeutet werden, dass die Sozialpädagog*innen in Bezug auf das Abschlussniveau auf Sekundarstufe II eher tiefe Erwartungen an die Jugendlichen haben, wie dies auch bereits Schaffner (2014) in einer anderen schweizerischen Studie aufgezeigt hat.

Bemerkenswert ist, dass alle befragten Jugendlichen Ideen für ihre zukünftige berufliche Entwicklung thematisieren, gleichzeitig aber auch Bedenken hinsichtlich der Realisierungschancen haben. Diese Äusserungen können entweder als Unsicherheit in Bezug auf die eigenen Fähigkeiten gewertet werden oder als Bewusstsein dafür, dass die Mittel zum Erreichen ihrer Ziele begrenzt sein könnten. Da die Unterstützung durch die Jugendhilfe spätestens mit Abschluss der Ausbildung endet und in der Schweiz keine Unterstützungsprogramme für Care Leaver existieren, ist es für sie auf Grund ihrer frühen finanziellen Selbstverantwortung und fehlender familiärer Unterstützung schwieriger, ihre beruflichen Ziele, z. B. eine zweite Ausbildung oder ein Studium, zu realisieren als für Gleichaltrige. Abschliessend ist vor diesem Hintergrund festzuhalten, dass in der Schweiz weitere Studien nötig sind, welche die berufliche Integration von Care Leavern längerfristig untersuchen und die gesellschaftliche Positionierung und Zufriedenheit der Care Leaver mit der eigenen biographischen Entwicklung beleuchten. Aus einer sozial- und bildungspolitischen Perspektive ist zu prüfen, mit welchen strukturellen und konzeptionellen Massnahmen, wie z. B. speziellen Programmen und Beratungsstellen, die Bildungschancen von Care Leavern in der Schweiz in Zukunft optimiert werden könnten.

\section{LITERATUR}

BFS Bundesamt für Statistik (2016): Newsletter Nr. 2 - Informationen aus der Demografie.

BFS Bundesamt für Statistik (2020): Übergang von der Sekundarstufe I in die Sekundarstufe II. Online verfügbar unter: https://www.bfs.admin.ch/bfs/de/home/statistiken/bevoelkerung/ migration-integration/integrationindikatoren/indikatoren/uebergang-sekundarstufe-|-II.html (09.02.2021).

Brady, Eavan/Gilligan, Robbie (2019): Exploring diversity in the educational pathways of care-experienced adults: findings from a life course study of education and care. In: Children and Youth Services Review, 104, o. S. 
Cameron, Claire/Hollingworth, Katie/Schoon, Ingrid/van Santen, Eric/Schröer, Wolfgang/Ristikari, Tiina/Heino, Tarja/Pekkarinen, Elina (2018): Care leavers in early adulthood: how do they fare in Britain, Finland and Germany? In: Children and Youth Services Review, 87, 163-172.

Courtney, Mark (2019): The Benefits of Extending State Care to Young Adults: Evidence from the United States of America. In: Mann-Feder, Varda/Goyette, Martin (Hrsg.): Leaving Care and the Transition to Adulthood. International Contributions to Theory, Research, and Practice. Oxford: Oxford University Press, 131-147.

Courtney, Mark/Dworsky, Amy/Lee, JoAnn/Raap, Melissa (2009): Midwest Evaluation of the Adult Functioning of Former Foster Youth: Outcomes at Age 23 and 24. Chicago: Chapin Hall at the University of Chicago.

Crain, Fitzgerald (2012): „Ich geh ins Heim und komm als Einstein heraus". Wiesbaden: VS Verlag für Sozialwissenschaften.

EDK Schweizerische Konferenz der kantonalen Erziehungsdirektoren (o. J.a): Bildungssystem Schweiz. Online verfügbar unter: https://www. edk.ch/dyn/14798.php (26.08.2020).

EDK Schweizerische Konferenz der kantonalen Erziehungsdirektoren (o. J.b): Bildungssystem. Online verfügbar unter: https://bildungssystem.educa.ch/de/sekundarstufe-ii-4 (26.08.2020).

Flick, Uwe (2012): Qualitative Sozialforschung. Reinbek bei Hamburg: Rowohlt.

Gilligan, Robbie/Arnau-Sabatés Laura (2017): The role of carers in supporting the progress of care leavers in the world of work. In: Child and Family Social Work, 22, 792-800.

Göbel, Sabrina/Hansmeyer, Annabell/Lunz, Marei/Peters, Ulla (2019): Occupational Aspirations of Care Leavers and their Pathways to Work. In: Social Work and Society, 17 (2), 1-20.

Groinig, Maria/Hagleitner, Wolfgang/Maran, Thomas/Sting, Stephan (2019): Bildung als Perspektive für Care Leaver? Bildungschancen und Bildungswege junger Erwachsener mit Kinderund Jugendhilfeerfahrung. Opladen: Verlag Barbara Budrich.
Hanrahan, Fidela/Boddy, Janet/Owen, Charlie (2020): 'Actually There is a Brain in There': Uncovering Complexity in Pathways Through Education for Young Adults Who Have Been in Care. In: Children \& Society, 34, 46-61.

Harder, Annemiek/Mann-Feder, Varda/Otterholm, Inger/Refaeli, Tehila (2020): Supporting transitions to adulthood for youth leaving care: consensus based principles. In: Children and Youth Services Review, 116.

Kompetenzzentrum Leaving Care (2020): Argumentarium für die Thematik Leaving Care. Bern. Online verfügbar unter: https://leaving-care.ch/ argumentarium-leaving-care (31.08.2020).

Köngeter, Stefan/Mangold, Katharina/Strahl, Benjamin (2016): Bildung zwischen Heimerziehung und Schule. Ein vergessener Zusammenhang. Weinheim: Beltz Juventa.

Kuckartz, Udo (2012). Qualitative Inhaltsanalyse. Methoden, Praxis, Computerunterstützung. Weinheim: Beltz Juventa.

Mangold, Katharina/Rein, Angela (2014): Formale Bildung als Ressource für Care Leaver in Übergängen ins Erwachsensein. In: Forum Erziehungshilfen, 20 (3), 141-146.

Meyer, Thomas (2018): Von der Schule ins Erwachsenenleben: Ausbildung- und Erwerbsverläufe in der Schweiz. In: Social Change in Switzerland, 13.

O'Higgins, Aoife/Sebba, Judy/Luke, Nikki (2015): What is the relationship between being in care and the education outcomes of children? An international systematic review. Oxford: Rees Centre for Research in Fostering and Education.

Petrucci, Marco/Wirtz, Markus (2007). Sampling und Stichprobe. QUASUS. Qualitatives Methodenportal zur Qualitativen Sozial-, Unterrichtsund Schulforschung. Online verfügbar unter: https://quasussite.wordpress.com/samplingund-stichprobel (04.01.2021).

Reimer, Daniela (2017): Normalitätskonstruktionen in Biografien ehemaliger Pflegekinder. Weinheim: Beltz Juventa.

Rein, Angela (2020): Normalität und Subjektivierung. Eine biographische Untersuchung im Übergang aus der stationären Jugendhilfe. Bielefeld: transcript Verlag. 
Rogers, Justin (2017): 'Different' and 'Devalued': Managing the Stigma of Foster-Care with the Benefit of Peer Support. In: British Journal of Social Work, 47, 1078-1093.

Schaffner, Dorothee (2007): Junge Erwachsene zwischen Sozialhilfe und Arbeitsmarkt: biographische Bewältigung von diskontinuierlichen Bildungs- und Erwerbsverläufen. Bern: hep Verlag.

Schaffner, Dorothee (2014): Erziehung zu Anpassung und Unterordnung. Berufsintegration im Sonderschulheim. In: Karl, Ute (Hrsg.): Rationalitäten des Übergangs in Erwerbsarbeit. Weinheim: Beltz Juventa, 44-60.

Schaffner, Dorothee/Läber, Myriame (2017): Es muss mehr sein als Erziehung zur Anpassung und Unterordnung. In: Zeitschrift für Sozialpädagogik, 15 (4), 415-433.

Schaffner, Dorothee/Rein, Angela (2013): Jugendliche aus einem Sonderschulheim auf dem Weg in die Selbständigkeit - Übergänge und Verläufe. In: Piller, Edith M./Schnurr, Stefan (Hrsg.): Kinder- und Jugendhilfe in der Schweiz. Wiesbaden: Springer Fachmedien, 53-78.

Schaffner, Dorothee/Rein, Angela (2014): Strukturelle Rahmung der Statuspassage Leaving Care in der Schweiz - Sondierung in einem unübersichtlichen Feld. In: Schweizerische Zeitschrift für Soziale Arbeit, 16 (I), 9-26.

Schmidlin, Sabina/Kobelt, Emilienne/Caviezel, Urezza (2018): Reduktion der Abhängigkeit von Jugendlichen und jungen Erwachsenen in der Sozialhilfe. Forschungsbericht Nr. 7/18. Bern: Bundesamt für Sozialversicherungen.

Sievers, Britta/Thomas, Severine/Zeller, Maren (2015): Jugendhilfe - und dann? Zur Gestaltung der Übergänge junger Erwachsener aus stationären Erziehungshilfen. Frankfurt: IGfH Eigenverlag.

SKBF Schweizerische Koordinationsstelle für Bildungsforschung (2018). Bildungsbericht Schweiz 2018. Aarau: Schweizerische Koordinationsstelle für Bildungsforschung.
SBFI Staatssekretariat für Bildung, Forschung und Innovation SBFI (2020): Berufsbildung in der Schweiz. Fakten und Zahlen 2020. Bern. Online verfügbar unter: https://www.berufsbildung.ch/ dyn/5329.aspx (26.08.2020).

Stalder, Anita/Clerc, Isabelle (2018). Entwicklung eines Modells für die nachhaltige berufliche Grundbildung. In: Tokarski, Kim Oliver/Schellinger, Jochen/Berchtold, Philippe (Hrsg.): Nachhaltige Unternehmensführung. Herausforderungen in Beispiele aus der Praxis. Wiesbaden: Springer Gabler, 337-363.

Stein, Mike (2012): Young People Leaving Care. Supporting Pathways to Adulthood. London: Jessica Kingsley Publishers.

Stohler, Renate (2005): Nachuntersuchung Lernstatt Känguruh. Zürich: Universität Zürich.

Stohler, Renate/Gehrig, Milena (2014): Wohnen und Selbständigkeit. Institutionelle Unterstützung prekärer Übergänge ins Erwachsenenalter. In: Schweizerische Zeitschrift für Soziale Arbeit 16 (1), 94-109.

Strahl, Benjamin (2019): Heimerziehung als Chance? Erfolgreiche Schulverläufe im Kontext von stationären Erziehungshilfen. Weinheim: Beltz Juventa.

Truschkat, Inga/Kaiser, Manuela/Reinartz, Vera. (2005). Forschen nach Rezept? Anregungen zum praktischen Umgang mit der Grounded Theory in Qualifikationsarbeiten. In: Forum Qualitative Sozialforschung, 6 (2). Online verfügbar unter https://www.qualitative-research.net/index.php/fas/article/view/470/1007

(04.01.2021).

Werner, Karin (2019): Leben als Pflegekind. Die Perspektive jugendlicher Pflegekinder auf ihre Lebenssituation. Weinheim: Beltz Juventa.

Witzel, Andreas (2000): Das problemzentrierte Interview. In: Forum Qualitative Sozialforschung, 1 (1). Online verfügbar unter: https://www.qualitative-research.net/index.php/fqs/article/view/ 1132/2520 (24.09.2020). 


\section{Zu den Autor*innen}

Karin Werner ist Dozentin und Projektleiterin an der Zürcher Hochschule für angewandte Wissenschaften (ZHAW). Ihre Forschungsschwerpunkte sind Pflegekinderhilfe, Leaving Care und Gesundheitsförderung im Kontext Sozialer Arbeit.

Renate Stohler ist Dozentin und Projektleiterin an der Zürcher Hochschule für angewandte Wissenschaften (ZHAW). Ihre Forschungsschwerpunkte sind Leaving Care, Kinder- und Jugendhilfe und Soziale Arbeit im Kontext Schule.

\section{Kontakt}

Dr. Karin Werner

Institut für Kindheit, Jugend und Familie

Zürcher Hochschule für Angewandte Wissenschaften (ZHAW)

Pfingstweidstrasse 96

$\mathrm{CH}-8037$ Zürich

Tel: +41589348839

E-Mail: karin.werner@zhaw.ch

URL: https://www.zhaw.ch/de/ueber-uns/person/wekal

Renate Stohler, lic. phil.

Institut für Kindheit, Jugend und Familie

Zürcher Hochschule für Angewandte Wissenschaften (ZHAW)

Pfingstweidstrasse 96

$\mathrm{CH}-8037$ Zürich

Tel: +41589348867

E-Mail: renate.stohler@zhaw.ch

URL: https://www.zhaw.ch/de/ueber-uns/person/stol/ 